\title{
The influence of meteoroid rotation on the diffraction characteristics of underdense radio-meteors
}

\author{
G. G. Novikov ${ }^{1}$, P. Pecina ${ }^{2}$, and A. V. Ivanov ${ }^{1}$ \\ 1 Novgorod State University, Great Novgorod, Russia \\ 2 Astronomical Institute of the Academy of Sciences of the Czech Republic, 25165 Ondřejov, Czech Republic
}

Received 13 December 2001 / Accepted 2 December 2003

\begin{abstract}
We discuss the problem of meteor plasma formation and consequently of radio-wave scattering from an ionized meteor trails produced by rotating meteoroids. The problem is solved under the following approximations: the meteoroid is represented by a cube, its rotation axis is perpendicular to the velocity vector, meteoroid deceleration can be neglected, the only acting ablation mechanism is the evaporation, the deionization effects are negligibly small. In this case the ambipolar diffusion approximation applies, and, supposing that the source of free electrons is known, the distribution of free electrons inside a trail, $N_{\mathrm{e}}(\boldsymbol{r}, t)$, considered as a function of position and time, can be computed. It is shown that the electron distribution along a trail axis oscillates, which is not the case with respect to the radial distribution. The function $N_{\mathrm{e}}(\boldsymbol{r}, t)$ found is then used for the solution of the problem of the scattering of radio-waves on the underdense-type trails produced by rotating particles. The values for amplitude-time as well as phase-time characteristics computed under the above assumptions differ only very slightly from those computed when meteoroid rotation is not taken into consideration.
\end{abstract}

Key words. meteors, meteoroids

\section{Introduction}

The interest in the study of meteors has originated in astrophysics, geophysics and also in application of science to various practical problems. On the whole, the existing theory of meteoric phenomena describes the observational data on photographic and radio meteors rather well. However, some unsolved problems still remain. One such remaining problem is that of the influence of meteoroid rotation on the diffraction characteristics of underdense-type meteors. The aim of this work is to solve this problem.

The solution of this problem can depend upon the structure of meteoroids. It is known that most meteoroids (probably with the exception of Geminids) are not single monolithic particles (e.g. spheres, squares and so on), they are now considered to be best represented by dustballs composed of numerous small grains held together by some binding substance (e.g. Hawkes \& Jones 1978). This can make the solution of the rotation problem difficult since the irregular ablation expected can fully override the effect of their spinning. On the other hand, Geminids can probably be used for this study. At present it does not seem to be possible to find the solution of this problem for a body of any form and for a rotation axis making an angle $\theta$ to the velocity vector. Therefore, for the sake of illustration, we will assume that the shape of a meteoroid does not change during its flight in the atmosphere, but only its size, and that its initial shape is a cube with sides $a$. It is clear that if we obtain any effect for a cube then meteoroids of any shape should also show an observable effect. Since the cross-sectional area of a meteoroid will also change due to its rotation, this will lead to a variation of the ablation rate of neutral particles from the meteoroid surface along its trajectory and, consequently, to a variation of the electron concentration along the trail, and of the amplitude of the scattered signal. Rotation is inherent to all space bodies. This is documented by pulsations observed in meteor light curves (e.g. Kramer \& Shestaka 1983; Babadzhanov \& Konovalova 1987). Also Halliday (1988) reported that about $50 \%$ of Geminids observed with the MORP cameras showed flickering. Since Geminid meteoroids behave very much like single grains during their atmospheric flight they are suitable for studying the rotation effect. Moreover, dust particles in the solar system which are potential producers of meteoroids that are observed by radar have to spin, as reported by Misconi (1993) who provides a background of meteoroid spin-up mechanisms as well as estimates of meteoroid spin rates depending on their sizes. Other estimates were made by Hawkes \& Jones (1978), Olsson-Steel (1987), and Jones (1990). According to these authors meteoroid spinning can manifest itself in dimensions of the initial train radius larger than expected from theoretical computations. Jones (1990) treated the rotational damping of small interplanetary particles. We will use their estimated spin rates in Sect. 4 when computing the amplitude and phase-time characteristics. Other observable consequences of meteoroid rotation have been discussed by Beech \& Brown (2000) and Beech (2001). Even though these studies refer to fireballs, i.e. meteors caused by larger meteoroids, they may also be relevant in the case of fainter radio meteors. 
In the following sections we will first consider the problem of the time variability of a meteoroid cross-sectional area, then we will derive the expression for the electron volume density, and lastly we will compute the amplitude-time and phase-time characteristics.

\section{Variability of cross-sectional area}

We assume that a meteoroid has a cubic shape during its atmospheric flight. However, the size of the cube decreases due to ablation. Thus, the cross-sectional area will change both because of the rotation and due to ablation which we suppose to be isotropic. The ablation equation is (Bronshten 1983)

$\dot{m}=-(\Lambda / 2 Q) S \rho v^{3}$,

where $m$ stands for the instantaneous meteoroid mass, $v$ for its velocity at time $t, \Lambda$ is the heat transfer coefficient, $S$ designates the cross-sectional area of the meteoroid, $Q$ denotes the energy needed to ablate a unit of mass, and $\rho$ is the air density. We will assume that within the meteor height range we can put $\rho=\rho_{\mathrm{b}} \exp (t / T)$, where $T=H / v \cos z_{\mathrm{R}} ; H$ is the scale height, $\rho_{\mathrm{b}}$ the atmospheric density at the beginning height of the meteor, and $z_{\mathrm{R}}$ the zenith angle of the meteoroid radiant. We will also need the equation for the electron line density, $\alpha$. According to Bronshten (1983)

$\alpha v \mu=-\beta(v) \dot{m}$,

in which $\mu$ denotes the mass of an atom ablated from the meteoroid and $\beta=\beta_{0} v^{n}$ (with $n=3.5$ ) the ionization probability.

For simplicity we will assume that $\Lambda$ as well as $Q$ are constant during the meteoroid flight and that its deceleration is negligibly small. As already stated we also assume that the rotation axis is perpendicular to the meteoroid velocity vector. Taking into account how the cross-sectional area alters during one rotational period we can use the function

$S(t)=\frac{1}{2} a^{2}(t)[\sqrt{2}+1-(\sqrt{2}-1) \cos 4 \omega t]$,

describing the cross-sectional area variability caused by the meteoroid rotation characterized by its frequency, $\omega$, and by ablation represented by $a(t)$. Inserting Eq. (3) into Eq. (1) and bearing in mind that $m(t)=a^{3}(t) \delta$ where $\delta$ designates the meteoroid bulk density we arrive at the following expression for $a(t)$ :

$a(t)=a(0)-\frac{\Lambda \rho_{b} v^{3}}{12 Q \delta} \int_{0}^{t} \mathrm{e}^{t^{\prime} / T}\left[\sqrt{2}+1-(\sqrt{2}-1) \cos 4 \omega t t^{\prime} \mathrm{d} t^{\prime}\right.$,

where $a(0)=\left(m_{0} / \delta\right)^{1 / 3}$ is the initial size of the cube edge. The time dependence of $\alpha$ can be computed from

$\alpha(t)=(\Lambda / 2 \mu Q) \beta(v) v^{2} S(t) \rho(t)$,

following from Eqs. (1) and (2). The function $S(t)$ is given by Eq. (3) in which $a(t)$ must be computed in agreement with Eq. (4). The integration can be performed analytically, and the final formula for the electron line density reads

$$
\begin{aligned}
& \alpha(t, \omega)=(\Lambda / 2 \mu Q) \beta v^{2} \rho_{\mathrm{b}}[\sqrt{2}+1-(\sqrt{2}-1) \cos 4 \omega t] \mathrm{e}^{t / T} \\
& \quad \times\left\{a(0)-\left(\Lambda \rho_{\mathrm{b}} v^{3} T / 12 Q \delta\right)\left[(\sqrt{2}+1)\left(\mathrm{e}^{t / T}-1\right)+(\sqrt{2}-1)\right.\right. \\
& \left.\left.\quad \times\left(1+[4 \omega T]^{2}\right)^{-1}\left(1-\mathrm{e}^{t / T}[\cos 4 \omega t+4 \omega T \sin 4 \omega t]\right)\right]\right\}^{2} / 2 .
\end{aligned}
$$

\section{The electron volume density}

Since the dynamics of meteor plasma are determined in principle by the collisions between charged particles and neutral atmospheric species the formation and decay of the plasma can be described well within the ambipolar diffusion approximation with a given source of electrons. The source can be considered as discrete. We know that deionization processes (such as recombination, attachment, and so on) can be neglected for the underdense trails which are what we mostly observe by radars.

To solve the problem of the evolution of the volume density of free electrons inside the trail we formally introduce two diffusion coefficients: the first, $D_{\perp}$, characterizing the diffusion of meteor plasma in direction perpendicular to the trail axis, and the second, $D_{\|}$, characterizing the diffusion along the trail axis. The coordinate system is chosen as follows: the $z$-axis points along the velocity vector of the body and $x$ and $y$-axes are perpendicular to the $z$-axis so that $r=\sqrt{x^{2}+y^{2}}$ denotes the radius of the trail. Within the ambipolar diffusion approximation, the equation describing the variation of the electron density, $N_{\mathrm{e}}(x, y, z, t)$, with the known source, $S_{\mathrm{e}}$, reads:

$\frac{\partial N_{\mathrm{e}}}{\partial t}-D_{\perp}\left(\frac{\partial^{2} N_{\mathrm{e}}}{\partial x^{2}}+\frac{\partial^{2} N_{\mathrm{e}}}{\partial y^{2}}\right)-D_{\|} \frac{\partial^{2} N_{\mathrm{e}}}{\partial z^{2}}=S_{\mathrm{e}}$

where

$S_{\mathrm{e}}(x, y, z, t)=-(\beta / \mu)(\mathrm{d} m / \mathrm{d} t) \delta(x) \delta(y) \delta(z-v t)$,

is the source of free electrons. In Eq. (8) $\beta$ is the ionization probability due to atoms or molecules ablated from the meteoroid surface. The mass of these species is $\mu$ and $\delta($.$) is$ Dirac's function. The solution of Eq. (7) with the initial condition $N_{\mathrm{e}}(x, y, z, 0)=0$ can be written as:

$$
\begin{aligned}
N_{\mathrm{e}}(x, y, z, t)= & \frac{1}{8 \pi\left(D_{\perp}+D_{\|}\right)^{3 / 2}} \int_{0}^{t} \frac{G\left(t^{\prime}\right)}{\left(t-t^{\prime}\right)^{3 / 2}} \\
& \times \exp \left\{-\frac{x^{2}+y^{2}}{4 D_{\perp}\left(t-t^{\prime}\right)}-\frac{\left(z-v t^{\prime}\right)^{2}}{4 D_{\|}\left(t-t^{\prime}\right)}\right\} \mathrm{d} t^{\prime},
\end{aligned}
$$

where $G(t)=-(\beta / \mu)(\mathrm{d} m / \mathrm{d} t)$ is the source efficiency. However, the solution of Eq. (9) cannot be expressed by means of elementary functions. Therefore, Eq. (9) is too long to be useful in practice. Fortunately, it can be further simplified. Since the effective influence of the diffusion along the trail axis is negligibly small compared to the perpendicular diffusion (this effect is of the order $L_{\perp} / L_{\|}$where $L_{\perp}$ and $L_{\|}$are perpendicular and parallel dimensions of the meteor trail, respectively), then the limit $D_{\|} \rightarrow 0$ implies that

$\frac{1}{2 \pi \sqrt{D_{\|}\left(t-t^{\prime}\right)}} \exp \left\{-\frac{\left(z-v t^{\prime}\right)^{2}}{4 D_{\|}\left(t-t^{\prime}\right)}\right\} \rightarrow \delta\left(z-v t^{\prime}\right)$.

Integrating (9) with the above limit taken into account we arrive at

$N_{\mathrm{e}}(r, z, t)=\frac{\alpha(z / v)}{4 \pi D(t-z / v)} \exp \left\{-\frac{r^{2}}{4 D(t-z / v)}\right\}$,

where $\alpha(z / v)=-(\beta v / \mu)(\mathrm{d} m / \mathrm{d} z)$ is the electron line density.

The variation of electron volume density along the meteoroid path is given by the corresponding variation of the electron line density, $\alpha$. Figure 1 shows such a variation. It can be 


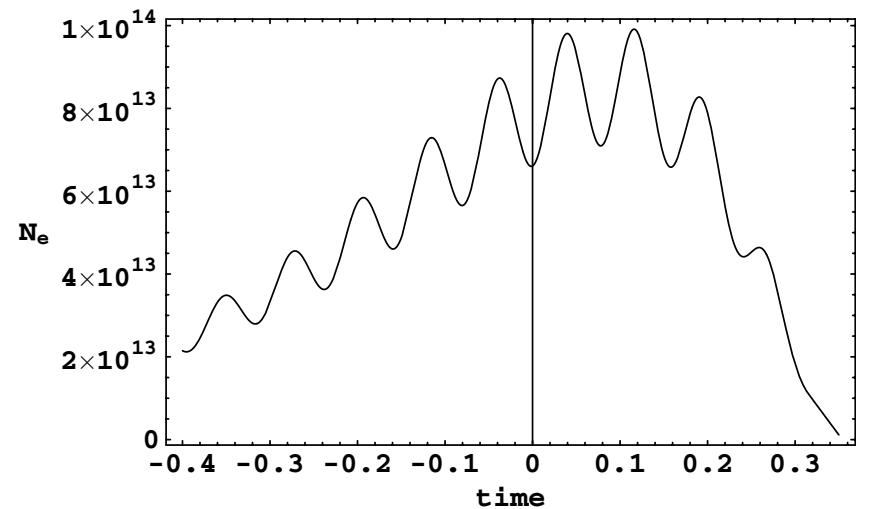

Fig. 1. Variation of the electron volume density, $N_{\mathrm{e}}\left(\right.$ in $^{-3}$ ), along the trail axis expressed as a function of time in seconds. The time is measured relative to the instant of the meteoroid passage of the specular point as explained in Sect. 4.

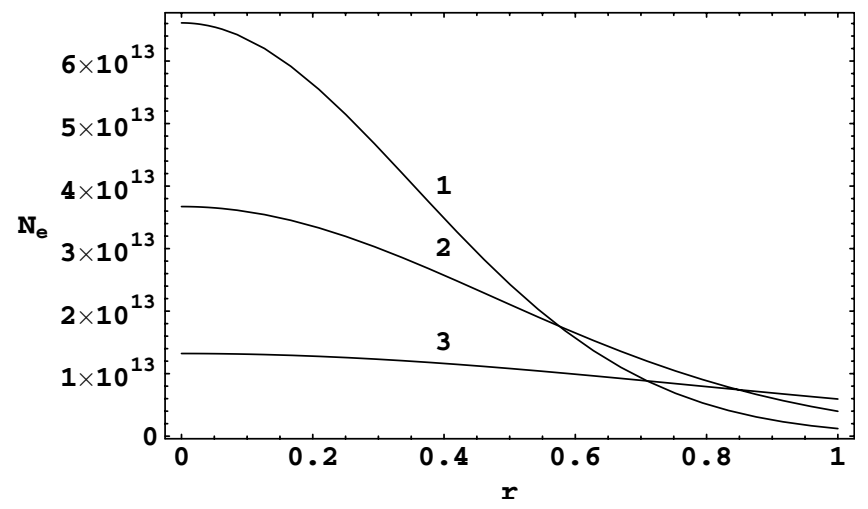

Fig. 2. Variation of the electron volume density, $N_{\mathrm{e}}$ (in $\mathrm{m}^{-3}$ ), at $z=0$ along the trail radius (in meters) for three different instants if time: $1-t=0 \mathrm{~s}, 2-t=0.01 \mathrm{~s}, 3-t=0.05 \mathrm{~s}$.

seen that taking the rotation of the body into account leads to fluctuations of $N_{\mathrm{e}}$ along the meteoroid trajectory. Figure 2 illustrates the variation of $N_{\mathrm{e}}$ as a function of $r$ at $z=0$ (i.e. at the specular point) for some instants of time $t$. For the construction of these two figures, the meteoroid parameters were chosen as follows: $Q=8 \times 10^{6} \mathrm{~J} \mathrm{~kg}^{-1}, \mu=3.8 \times 10^{-26} \mathrm{~kg}$, the initial mass of the meteoroid $m_{0}=5 \times 10^{-7} \mathrm{~kg}, H=6 \mathrm{~km}, v=40 \mathrm{~km} \mathrm{~s}^{-1}$, $\Lambda=1, \cos z_{R}=0.6, \delta=3500 \mathrm{~kg} \mathrm{~m}^{-3}, h_{\mathrm{b}}=95 \mathrm{~km}$, and $D=5 \mathrm{~m}^{2} \mathrm{~s}^{-1}$.

In the next section we will use Eq. (10) to compute both the amplitude-time and phase-time characteristics.

\section{Amplitude-time and phase-time characteristics}

Now let us apply the preceding results to the task of clarifying the influence of the rotation of the body on the character of the reflection of radio waves from the trails. We will study the behaviour of the amplitude-time (or Fresnel, hereafter as FCH) and phase-time $(\mathrm{PCH})$ characteristics of the signal scattered from the trails of underdense types. In the case of such trails it is known that each electron scatters the incident wave independently of any other electron, and that the scattering is coherent (e.g. McKinley 1961; Kashcheev et al. 1967; Lebedinets 1980). The radar equation reads in this case (e.g. Novikov et al. 1986)

$P_{\mathrm{R}}=P_{\mathrm{T}} \frac{G_{\mathrm{T}} G_{\mathrm{R}} r_{\mathrm{e}}^{2} \lambda^{2}}{16 \pi^{2}}\left|\int_{V_{\mathrm{e}}} \frac{N_{\mathrm{e}}}{R^{2}} \mathrm{e}^{\mathrm{i} 4 \pi R / \lambda} \mathrm{d} V_{\mathrm{e}}\right|^{2}$,

where $P_{\mathrm{T}}$ is the power transmitted by a radar, $G_{\mathrm{T}}$ and $G_{\mathrm{R}}$ are the gain of the transmitting and receiving antennae, respectively, $r_{\mathrm{e}}$ is the classical radius of the electron, $\lambda$ is the wavelength of the transmitted wave, $V_{\mathrm{e}}$ stands for the scattering volume $\left(\mathrm{d} V_{\mathrm{e}}=r \mathrm{~d} r \mathrm{~d} \varphi \mathrm{d} z\right)$, and $R$ is the distance of the scattering point from an observer. It will be advantageous to locate the origin of the frame of reference into the specular point of the trail. Then the $x$-axis points to the observer (i.e. the radar) which is at a distance $R_{0}$. Since the principal contribution to the received signal comes from the region around the specular point we can write

$R \simeq R_{0}+r \cos \varphi+z^{2} / 2 R_{0}$,

where $r$ is the radial polar coordinate while $\varphi$ is the corresponding azimuthal angle. The $z$-coordinate is measured along the trail axis positively in the direction of meteoroid motion, and $z=0$ at the specular point. The meteoroid passage of this point corresponds to $t=0 \mathrm{~s}$. In order to simplify our formulae and not be forced to calculate with numbers of the order of $10^{12}$ or so we use in Eq. (10) $\alpha_{r}(t, \omega)=\alpha(t, \omega) / \alpha(0,0)$ instead of $\alpha(t, \omega)$. When substituting the expression (10) into (11), and making some simple transformations, we get the following formula for the power of the signal received from the underdensetype trail formed by a rotating meteoroid:

$P_{\mathrm{R}}=P_{\mathrm{T}} \frac{G_{\mathrm{T}} G_{\mathrm{R}} r_{\mathrm{e}}^{2} \lambda^{3}}{32 \pi^{2} R_{0}^{3}}|I|^{2}$,

where the quantity $I$ (generally complex) defined by the formula

$I=\frac{1}{\sqrt{2}} \int_{-\infty}^{x_{i}} \alpha_{r}(\omega, x) \exp \left\{\mathrm{i} \pi x^{2} / 2-\nabla\left(x-x_{i}\right)\right\} \mathrm{d} x$,

determines the diffraction characteristics. Here we have used the following notations:

$x_{i}=2 v t_{i} / \sqrt{R_{0} \lambda}, \quad x=2 z / \sqrt{R_{0} \lambda}, \quad \nabla=8 \pi^{2} D \sqrt{R_{0} \lambda} / v \lambda^{2}$.

Since the amplitude-time characteristics (i.e. FCH), $A_{i} \sim \sqrt{P_{\mathrm{T}}}$, it is clear that $A_{i}=|I|$. The phase angle of the received signal, $P_{i}$, can be computed from the ratio of imaginary to real part of $I$ as:

$\tan P_{i}=\frac{\int_{-\infty}^{x_{i}} \alpha_{r}(\omega, x) \exp \left\{-\nabla\left(x-x_{i}\right)\right\} \sin \left(\frac{\pi}{2} x^{2}\right) \mathrm{d} x}{\int_{-\infty}^{x_{i}} \alpha_{r}(\omega, x) \exp \left\{-\nabla\left(x-x_{i}\right)\right\} \cos \left(\frac{\pi}{2} x^{2}\right) \mathrm{d} x}$.

We have used $R_{0}=150 \mathrm{~km}$ and $\lambda=8 \mathrm{~m}$ during all our computations while $v$ has been the same as in the previous section. As already mentioned the value of the spin rate, $\omega$, was estimated by several authors to reach several hundred $\mathrm{Hz}$ (e.g. Hawkes \& Jones 1978; Olsson-Steel 1987; Jones 1990; Misconi 1993). To cover all the possible ranges from nonrotating cases to spin rates (say) $400 \mathrm{~Hz}$ we decided to compute our curves for $\omega=0,20,40,200$, and $400 \mathrm{~Hz}$. The curves valid for 


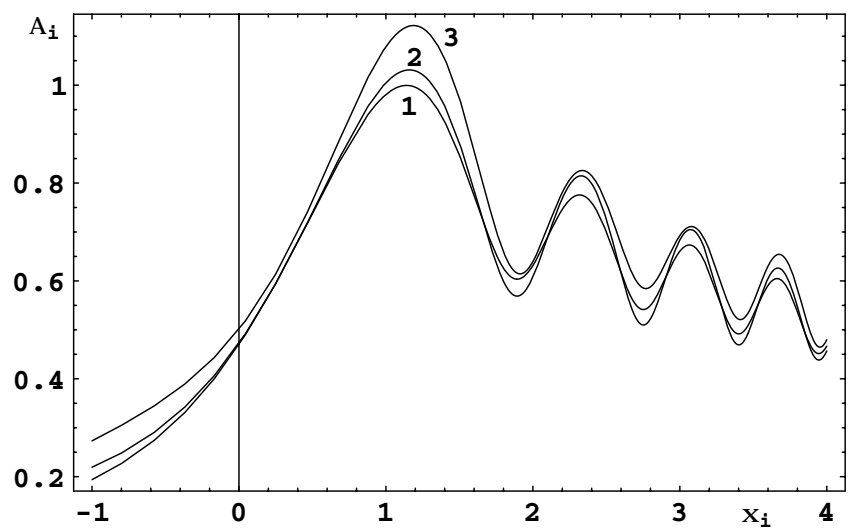

Fig. 3. Amplitude-time characteristics $(\mathrm{FCH})$ represented by the amplitude, $A_{i}$, as a function of $x_{i}$ for the following values of $\omega: 1$ $\omega=0 \mathrm{~Hz}, 2-\omega=20 \mathrm{~Hz}, 3-\omega=40 \mathrm{~Hz}$.

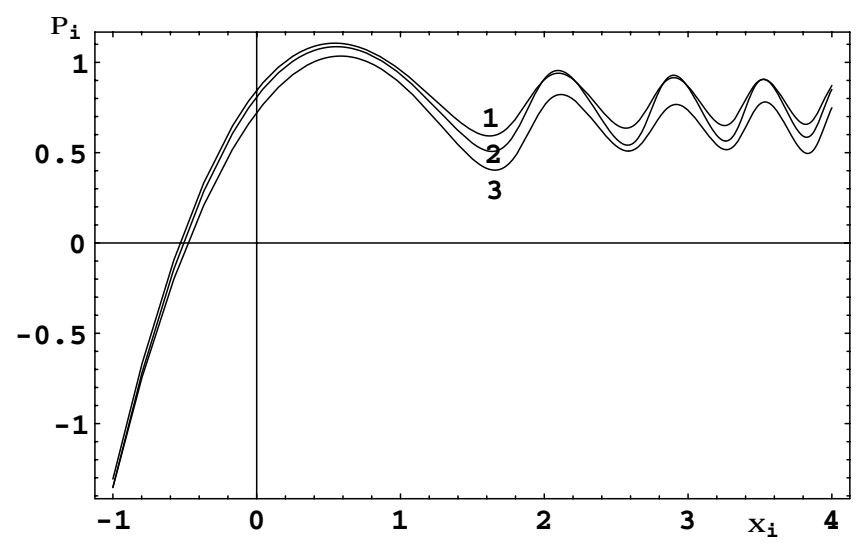

Fig. 4. Phase-time characteristics $(\mathrm{PCH})$ represented by the phase angle, $P_{i}$, in radians as a function of $x_{i}$ for the following values of $\omega$ : $1-\omega=0 \mathrm{~Hz}, 2-\omega=20 \mathrm{~Hz}, 3-\omega=40 \mathrm{~Hz}$.

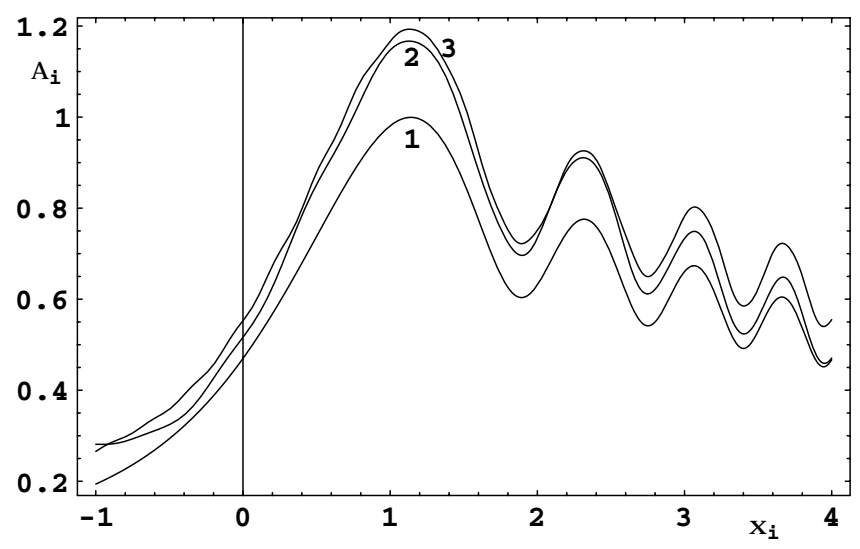

Fig. 5. The same as in Fig. 3 but for: $1-\omega=0 \mathrm{~Hz}, 2-\omega=200 \mathrm{~Hz}$, $3-\omega=400 \mathrm{~Hz}$.

$\omega=20$ and 40 are presented in Fig. 3 which shows FCH while Fig. 4 shows the analogous view for $\mathrm{PCH}$. The curves valid for $\omega=200$ and $400 \mathrm{~Hz}$ are shown in Figs. 5 and 6. All these curves are compared to the curve of a nonrotating meteoroid.

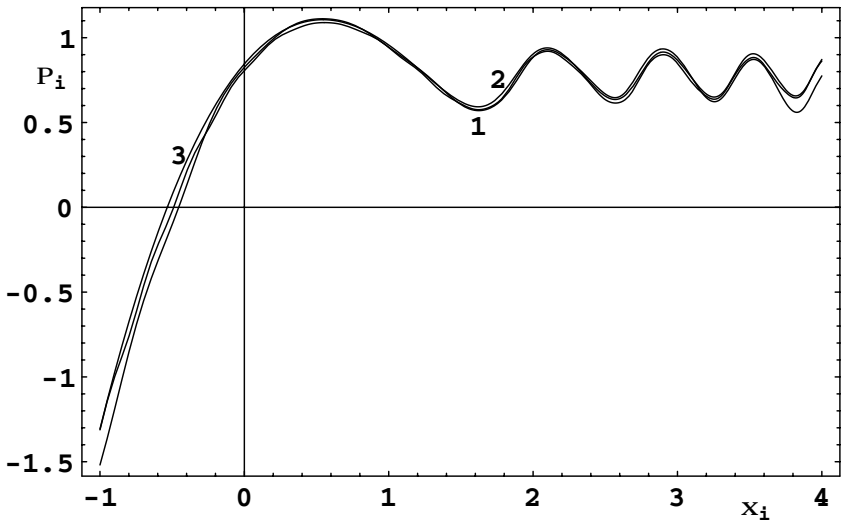

Fig. 6. The same as in Fig. 4 but for: $1-\omega=0 \mathrm{~Hz}, 2-\omega=200 \mathrm{~Hz}$, $3-\omega=400 \mathrm{~Hz}$

\section{Conclusion}

All Figs. 3-6 clearly indicate that the positions of maxima as well as minima of FCH and PCH change only very little due to the rotation of the meteoroid. This implies that the omission of rotation does not influence the velocity determination of the parent body within the model we have adopted. On the other hand, the consideration of rotation can change the magnitude of the diffraction maxima and minima which could manifest itself in the determination of the ambipolar diffusion coefficient when using $\mathrm{FCH}$ or $\mathrm{PCH}$.

Acknowledgements. The second author's contribution has been supported by the key project K3012103. The authors would like to express their thanks to Dr. M. Beech for his helpful advice. The authors also appreciate the help of Dr. J. Katgert with the correction of the wording of the article.

\section{References}

Babadzhanov, P. B., \& Konovalova, N. A. 1987, On the light pulsation of bright Geminids according to photographic data, Proc. 10th European regional astronomy meeting of the IAU, Praha, Czechoslovakia, ed. Z. Ceplecha, \& P. Pecina, Astronomical Institute of the Czechoslovak Academy of Sciences, Ondřejov, Czechoslovakia, 189

Beech, M. 2001, MNRAS, 326, 937

Beech, M., \& Brown, P. 2000, Planet. Space Sci., 48, 925

Bronshten, V. A. 1983 Physics of Meteoric Phenomena (Dordrecht, Boston, Lancaster: Kluwer acad. publ.)

Halliday, I. 1988, Icarus, 76, 279

Hawkes, R. L., \& Jones, J. 1978, MNRAS, 185, 727

Jones, W. 1990, MNRAS, 247, 257

Kashcheev, B. L., Lebedinets, V. N., \& Lagutin, M. F. 1967, Rezul'taty Issled. IGY, Issled. Meteorov No. 2

Kramer, E. N., \& Shestaka, I. S. 1983, Meteornaya Materiya v Atmosfere Zemli i Okolosol'nechnom Kosmicheskom Prostranstve (Moscow: Nauka)

Lebedinets, V. N. 1980, Pyl' v Verchnej Atmosfere i Kosmicheskom Prostranstve. Meteory. (Moscow: Gidrometeoizdat)

McKinley, D. W. R. 1961, Meteor Science and Engineering (New York: McGraw Hill)

Misconi, N. 1993, J. Geophys. Res., 98 (A11), 18951

Novikov, G. G., Pecina, P., \& Blokhin, A. V. 1986, Bull. Astron. Inst. Czechosl., 37, 189

Olsson-Steel, D. 1987, MNRAS, 226, 1 